\title{
Research on Network Coding Theory in Galois Domain Based on Energy Efficiency
}

\author{
Ren $\mathrm{Gao}^{1 *}$, Zhou $\mathrm{He}^{1}$, Chao $\mathrm{Pan}^{1}$, Ruoshan Kong ${ }^{2}$ \\ ${ }^{1}$ School of Information and Communication Engineering, Hubei University of Economics, Wuhan, 430072 China \\ ${ }^{2}$ School of Computer Science, Wuhan University, Wuhan, 430072 China \\ Email: gr@hbue.edu.cn
}

\begin{abstract}
With the development of network coding technology and network information theory, combining network coding theory with energy efficiency routing technology has become a hot topic in wireless networks. This paper studies the development of network coding technology and its present situation at domestic and abroad, the construction method of network coding in Galois domain is analyzed.
\end{abstract}

Keywords: Galois domain, network coding, energy efficiency

\section{Introduction}

Network coding is one of the key technologies in the wireless communication networks. The most intuitive understanding is that nodes in the network add the functions of encoding and decoding. Network information is effectively processed by encoding in nodes, then the processed data are transferred or transmitted. Network coding can be very useful in achieving a balance between energy efficiency and end-to-end packet error, improving the utilization of spectrum resources, particularly if only a subset of the nodes act as encoding nodes. It can transmit more data within limited spectrum resources, greatly improve the transmission capacity of communication channel and save network bandwidth.

\section{Development of Network Coding Technology and Its Present Situation}

\subsection{Development of Network Coding Technology}

Network coding was initially introduced in "Network Information Flow Theory" ${ }^{[1]}$ in 1998. In 2000, it is formally published by R. Ahlswede, N. Cai, S. Y. R. Li and R. W. Yeung in the paper "Network Information Flow [2]" on the IEEE Transactions on Information Theory. This is the foundation study of network coding, network coding and routing technology are integrated for the first time, and a new data packet transmission system is established. It is theoretically proved that if the information transmitted by intermediate nodes is not only limited to storage and forward, but encoded in an appropriate way, the system can achieve the maximum transmission rate in theory. Since then, many scholars have published a number of important research papers in this field, which have milestone significance.

(1) "Linear Network Coding" ${ }^{33]}$ published by Prof. Li shuoyan is pointed out that the network capacity in multicast mode can be achieved by using linear network coding technology. This paper won the best paper award of the IEEE Information Theory Society in 2005.

(2) Koetter and Edard et al. ${ }^{[4]}$ proposed an algebraic framework based on network coding, which uses abstract algebra to solve the problems of linear network coding. This idea provides a powerful and effective mathematical tool for the study of network coding. These researches further improve and enrich the network coding theory.

(3) In October 2012, the news that "coding TCP makes wireless networks 10 times faster" made a greater sensation in academia. A team of experts from MIT, Caltech/CIT, Harvard and some European universities improved wireless network transmission speed more than 10 times on October 13, 2012 without changing transmit power, network structure and transmission bandwidth.

(4) In 2016, the NetCoDer scheme is proposed to improve the communication reliability. It is based on merging cooperative diversity techniques and network coding. The obtained results highlight that the 
TDMA-based (Time Division Multiple Access) retransmission techniques: BlockACK, Master/Slave and Redundant TDMA. ${ }^{[5]}$

(5) ImadEz-zazi te al., investigated the trade-off between reliability and energy efficiency and proposed adaptive FEC/FWD and FEC/ARQ coding frameworks for clustered WSNs in 2017 $7^{[6]}$. Simulation results show that both the proposed frameworks are energy efficient compared to ARQ schemes and FEC schemes, and suitable to prolong the clustered network lifespan as well as improve the reliability.

(6) In the article of "Design of network coding based reliable sensor networks" in 2019 ${ }^{[7]}$, a mathematical model and a heuristic algorithm are proposed to plan for the best placement of encoding nodes while ensuring reliability. The experimental results show that the adequate number and placement of encoding nodes can be effectively determined, significantly enhancing the performance of constrained sensor networks using network coding.

Network coding completely overthrows the traditional way of information processing in communication network, realizes the maximum transmission rate, and has epoch-making significance in the field of information theory.

\subsection{Basic Ideas of Network Coding}

The basic principle of network coding is explained by the classical butterfly network as an example. As the network $G=(V, E)$ shown in fig. $1, V$ is the node set, $E$ is the edge set, $S$ is the source node, $T_{1}$ and $T_{2}$ are the destination nodes, and the other nodes are the intermediate nodes. Source node $\mathrm{S}$ sends the messages of $a$ and $b$ to both destination nodes $T_{1}$ and $T_{2}$.

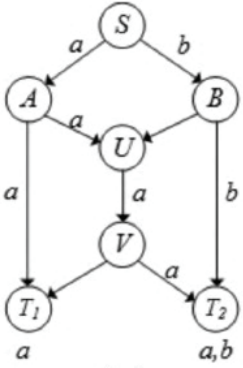

(a)

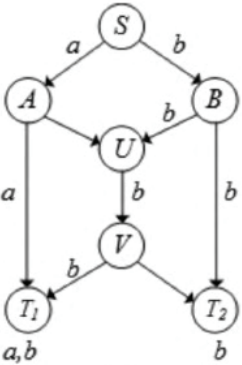

(b)

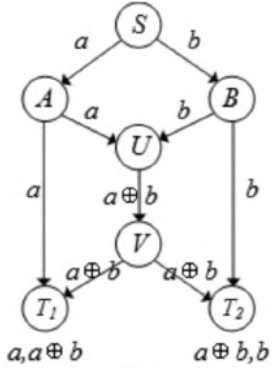

(c)

Figure 1. Basic ideas of network coding

Fig. 1 (a) and (b) are both traditional routing methods for data transmission, (c) adopts network coding method for data transmission. Because channel capacity is a unit capacity, it is easy to find that the traditional routing way can only choose to send $a$ or $b$ in the node $U$, and only one node $T_{1}$ or $T_{2}$ can receive data $a$ and $b$ at the same time. If the network coding is adopted, the node $U$ do $a \oplus b$ and then sent the result of $a \oplus b$ to the next node. When $T_{1}$ receives $a$ and $a \oplus b$, it can get $B$ by $a \oplus a \oplus b=b$. Similarly, $T_{2}$ can also get $a$, so $T_{1}$ and $T_{2}$ can receive $a$ and $b$ simultaneously. This is the original network coding theory, which can achieve the maximum flow of the network.

\section{Construction of Network Coding in Galois Domain}

\subsection{Basic Principle for Galois}

The core of Galois theory ${ }^{[8]}$ is to establish a one-to-one correspondence between intermediate domains set of domain extension $E / F$ and subgroups set of Galois group $G a K E / F$. This correspondence is counter-ordered and keeps commonality. $E / F$ is finite Galois expansion, that is, $E / F$ is finite separable normal expansion. Through this correspondence, a series of difficult problems, such as the root solvability of polynomial equation, are well solved by using the transformation between domain and group and the study of complementarity. 


\subsection{Node Energy Consumption Model for Wireless Networks}

We integrate the network coding theory into the energy consumption model of wireless networks and energy-saving routing technologies. Its energy consumption model can be defined as follows:

If the scope of network is $A=L^{*} L$, the transmission radius of the nodes is $r$, the energy consumption model between two nodes can be expressed as follows:

$$
E=c * v * d^{2}
$$

$c$ is a constant coefficient, $v$ is the transmission rate (bits/sec, bps), and $d$ is the rdistance between two nodes.

$E_{i}(0)$ stands for the original battery energy level when time $t=0$ for node $i$. Considering all energy consumption, the remaining battery energy $E_{i}(i)$ at time $t$ will satisfy:

$$
\begin{aligned}
E_{i}(t) & =E_{i}(0)-\int_{0}^{t} P_{i}(\tau) \mathrm{d} \tau-p^{C_{t}} \\
& =E_{i}(0)-\int_{0}^{t} P_{i}^{R F}(\tau) \mathrm{d} \tau-p^{T} \int_{0}^{t} I\left\{P_{i}^{R F}(\tau)>0\right\} \mathrm{d} \tau-p^{R} \sum_{j \in N-\{i\}} \int_{0}^{t} I\left\{d_{i j} \leq R_{j}(\tau)\right\} \mathrm{d} \tau-p^{C_{t}}
\end{aligned}
$$

$\int_{0}^{t} I\left\{P_{i}^{R F}(\tau)>0\right\} \mathrm{d} \tau$ is the period length during node $i$ is working in the time $t, \int_{0}^{t} I\left\{d_{i j} \leq R_{j}(\tau)\right\} \mathrm{d} \tau$ is transmission period length from node $i$ to node $j$.

\subsection{Node Network Coding Scheme for Wireless Networks}

Figure 2 is a wireless network with five nodes. In this mechanism, assuming that wireless node $A$ needs to transmit packet 1 to node $C$ and $D$, node $B$ needs to transmit packet 2 to node $C$ and $D$, and node $C$ is within the transmission range of node $A$, node $C$ can receive packet 1 directly, Similarly, node $D$ can receive packet 2 directly. Node $S$ needs to forward packet 1 to node $D$ and packet 2 to node $C$.

If without network coding mechanism, four transfers are required at least: a) node $A$ sends packet 1 to node $C$ and $S$; b) node $B$ sends packet 2 to node $D$ and $S$; c) node $S$ sends packet 1 to node $D$; d) node $S$ sends packet 2 to node $C$.

If network coding mechanism is adopted, three transfers are required: a) node $A$ sends packet 1 to node $C$ and $S$; b) node $B$ sends packet 2 to node $D$ and $S$; c) node $S$ sends network coding packet $1 \oplus$ packet 2 to node $C$ and $D$. In this case, network coding can reduce packet transmission rate by $2: 5 \%$, and consequently reduce energy consumption.

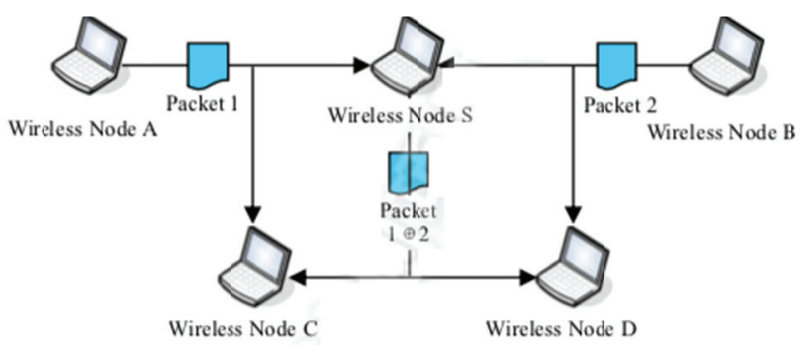

Figure 2. Node network coding scheme

\subsection{Data Packet Coding Mechanism for Nodes}

(1) Network model: acyclic communication network can be expressed as directed graph $G(V, E), V$ as node set, $E$ as link set, the input link of node $V$ as In $(V)$, and the output link as Out $(V)$.

For any pair of nodes $(d, e), x_{d e}$ represents data streams for $(d, e)$; if they are not adjacent, then $k_{d, e}=0$, $k_{d, e}$ is encoding coefficient, $S$ is source node. $K_{C}$ is $|E| \times|E|$ matrix. $P$ represents major ideal domains, $\omega$ is dimension of $G . J_{\omega,|E|}$ is $\omega \times|E|$ matrix.

(2) Network coding model: For source node $S$, the p-disjoint path set $P$ between source node $S$ and destination node $T$ is constructed. For the $\omega$-dimensional column vector $f_{e}$ of each edge $e$ on $P$ :

$$
f_{e}=\sum k_{d, e} f_{d}, v \text { is not a source node, } e \in \operatorname{Out}(v)
$$

Or similar descriptions: 


$$
\begin{gathered}
{\left[f_{e}\right]_{e \in E} \cdot\left(I_{|E|}-K_{C}\right)=J_{\omega,|E|}} \\
\operatorname{Det}\left(I_{|E|}-K_{C}\right)\left[f_{e}\right]_{e \in E}=J_{\omega,|E|} \bullet A d j\left(I_{|E|}-K_{C}\right)
\end{gathered}
$$

When solving $f_{e}$, it is necessary to ensure that there is a set of $C_{R}$ containing $P$ links for any receiving node $R \in T$, and the global coding kernel $G_{t}=\left\{f(e) \mid e \in C_{R}\right\}$ of this $p$ link is the basis of $K_{C}$.

\subsection{Packet Decoding Mechanism of Nodes}

In the decoding process, assuming that the node receives the network coding packet $P(g, y)$, there exists an $N \times N$ linear combination coding matrix $G, G X=Y . G_{i}$ denotes the row vector of $G, G_{i, j}$ is the elements of the $i$-row $j$-column of $G, Y$ is the $N \times 1$ linear combination coding vector, $X$ is the vector of $N \times 1$ which containing the symbol $x_{i}$. The switched Gauss elimination method is used in the packet decoding process. There are mainly two stages: triangulation and diagonalization.

Triangulation process: Each time the receiving node receives a new packet $P(g, y), g_{s}$ represents the first non-zero element of $G$. If $G_{s}$ is empty, the g vector is inserted in the s row of $G$, Otherwise, $g$ and $G_{s}$ are exchanged. The matrix $G$ is also called the upper triangular window matrix. This switch increases the probability of combined decoding and reduces the complexity of decoding.

Theorem: If the packet $P(g, y)$ is decoded by switched Gauss elimination, then $G$ is an upper triangular window matrix $\left(G_{i, j}=0\right.$, if $j<i$ or $\left.j>i+\omega-1\right)$, and $G_{i}$ is a coding vector of $P\left(G_{i}, Y_{i}\right)$.

Diagonalization process: When $N$ linearly independent packets are received, the rank of matrix $G$ equals $N$. Matrix diagonalization can be achieved by iterative XOR operations.

\section{Conclusions}

In recent years, network coding technology has become a hot technology because of its advantages of saving network resources consumption, improving spectrum resource utilization, greatly improving the transmission capacity of communication channels and saving network bandwidth. The combination of network coding theory and energy efficiency is a prospective research topic in the wireless networks. The research progress and current situation of network coding theory are studied in this paper. The construction method of network coding based on Galois domain is studied, the node energy consumption model of wireless network is constructed, and the encoding and decoding mechanism of nodes in wireless network is analyzed.

Acknowledgements. The work of this paper is supported by Natural Science Fund of Hubei Province (2017CFB773).

\section{References}

1. Ahlswede R,Cai N,Yeung R W. Network Information Theory[C], IEEE ISIT,1998.

2. Ahlswede R C N S Y. Network Information Flow[J]. IEEE Transactions On Information Theory. 2000,4(46): 1204-1216.

3. Li S Y R, Yeung R W, Cai N. Linear Network Coding[J], IEEE Transactions on Information Theory, 2003,49(2): 371-381.

4. Koetter R,Medard M. An algebraic approach to network coding[J]. IEEE/ACM Transactions on Networking. 2003,11(5): 782-795.

5. O.T. Valle, C. Montez, G. Medeiros de Araujo, F. Vasques, R. Moraes, Netcoder: A Retransmission mechanism for WSNs based on cooperative relays and network coding, Sensors 16 (6) (2016) 799.

6. I. Ez-zazi, M. Arioua, A. El Oualkadi, P. Lorenz, On the performance of adap- tive coding schemes for energy efficient and reliable clustered wireless sensor networks, Ad Hoc Netw. 64 (2017) 99-111.

7. E. AL-Hawri, N. Correia, A. Barradas. Design of network coding based reliable sensor networks $[\mathrm{J}]$. Ad Hoc Networks 91 (2019) 101870

8. Atin. Galois Theory [M]. Harbin: Harbin Institute of Technology Press (2011). 